The data and hospital records of 300 consecutive patients who underwent MILR (241 pure laparoscopic resections, 32 hand-assisted laparoscopic resections and 27 laparoscopyassisted open resections) were retrospectively reviewed and compared with data and hospital records of 100 matched patients who underwent open liver resection during the same period. The types of MILR performed included segmentectomy, bisegmentectomy, left and right hepatectomy, right trisectionectomy and caudate lobectomy for either benign $(n=197$, including 20 live-donor right lobectomies) or malignant $(n=103)$ etiologies.

Compared with open resection, patients who underwent MILR experienced shorter operative times (mean 99 mins vs 182 mins), less intraoperaterive blood loss (mean $102 \mathrm{ml}$ vs $325 \mathrm{ml}$ ), reduced need for transfusion (2 of 300 patients vs 8 of 100 patients), shorter hospital stays (mean 1.9 days vs 5.4 days), fewer overall operative complications ( $9.3 \%$ vs $22 \%$ ) and lower frequency of local malignancy recurrence ( $2 \%$ vs $3 \%$ ). No laparoscopic or hand-assisted laparoscopic resections required conversion to open resection, although two laparoscopyassisted open procedures required conversion to a fully open approach. Cost analysis suggested that MILR might be financially preferable to open procedures.

The authors conclude that MILR compares favorably with open resection for treating patients with benign and malignant hepatic lesions.

Original article Koffron AJ et al. (2007) Evaluation of 300 minimally invasive liver resections at a single institution: less is more. Ann Surg 246: 385-394

\section{Angiogenesis occurs at the onset of the dysplastic stage of colorectal cancer development}

In addition to its role as a key initiator of blood coagulation, tissue factor (TF) also upregulates vascular endothelial growth factor (VEGF), a highly potent stimulator of angiogenesis. The stage at which angiogenesis begins (the 'angiogenic switch') in the adenoma-carcinoma sequence (ACS) of colorectal cancer progression, however, is not clear. Staton and colleagues therefore examined the expression of TF and VEGF and microvascular density at different stages of colorectal cancer.
Historical surgical specimens from 210 patients were histopathologically examined; these specimens represented a range of colorectal cancer development stages, including background mucosa, low-grade and highgrade dysplastic polyps and Dukes' grade A, B and $C$ carcinoma. Fresh tissue specimens from 90 patients who underwent removal of primary colorectal cancer were also examined.

Microvascular density (measured using the cumulative Chalkley score) increased significantly with progression along the ACS $(P<0.0005)$, and was also positively correlated with the Dukes' stage $(P=0.013)$ and lymph node involvement $(P=0.023)$. The largest increase in the cumulative Chalkley score was observed at the onset of dysplasia. Semiquantitative analysis of TF and VEGF expression showed a positive correlation between the levels of these two markers of angiogenesis in the initial phase of the ACS $(P<0.005)$, but not with microvascular invasion, tumor size, Dukes' grade, lymph node involvement or survival.

The authors' findings suggest that the angiogenic switch occurs at the onset of dysplastic transformation of colorectal polyps, and that the correlation between TF and VEGF levels might highlight the importance of these markers in angiogenesis and disease progression.

Original article Staton CA et al. (2007) The angiogenic switch occurs at the adenoma stage of the adenomacarcinoma sequence in colorectal cancer. Gut 56: 1426-1432

\section{Chronic enterovirus infection might contribute to chronic fatigue syndrome development}

The precise cause of chronic fatigue syndrome (CFS) remains unknown, although infection by several viruses and bacterial species has been implicated in the etiology of this condition. As there is mounting evidence to suggest that enteroviruses might have a prominent pathogenic role in CFS, Chia and Chia tested for the presence of enterovirus in stomach biopsy specimens from 165 patients with CFS.

Antrum biopsy was performed in 165 consecutive patients with CFS; immunoperoxidase staining was used to detect viral capsid protein 1 , reverse-transcriptase polymerase chain reaction was used to test for enterovirus RNA, and viral culture was used to detect live virus. The results were compared with biopsies obtained 